\title{
Generalized Synchronization for Two New Different Chaotic Systems
}

\author{
Ping Zhou NianYing Zhang
}

Institute of Applied Physics, Chongqing University of Posts and Telecomm., Chongqing 400065, P. R. China

\begin{abstract}
One generalized synchronization method for two new chaotic systems is established and the mathematical proof of this method is provided. Theoretical analyze and simulation results show that the method in this paper is effective.
\end{abstract}

Keywords: Different chaotic system, Chaotic synchronization, Feedback control

\section{Introduction}

Chaotic synchronization and control have held many authors interest in the past few decades [1]-[13]. Two types of current concepts of synchronization are the identical synchronization and generalized synchronization. The identical synchronization means the case where states of two systems are equal or asymptotically equal as time goes to infinity. The generalized synchronization means the case where states of two systems satisfy a functional relation or asymptotically satisfy a functional relation as time goes to infinity. Chaotic generalized synchronization has attracted great interest due to its theoretical challenge and its great potential applications in secure communications, chemical reactions, and modeling brain activity.

Furthermore, more and more applications of chaos synchronization in secure communications make it much more important to synchronization two new different chaotic systems.

In this paper, we proposed a generalized synchronization method of two new different chaotic systems. Our method doesn't cancel all nonlinear information of response system. This is different with many investigated results [14]-[15]. Furthermore, we obtain the control law theoretically rigorous. Theoretical analyze and simulation results show that the method in this paper is effective.

\section{Generalized synchronization method between different chaotic systems}

Recently, two new chaotic systems have been reported [15]. They are the following system (1) and system (2).

$$
\begin{aligned}
& \left\{\begin{array}{l}
d x_{1} / d t=20 x_{1} / 7-x_{2} x_{3} \\
d x_{2} / d t=-10 x_{2}+x_{1} x_{3} \\
d x_{3} / d t=-4 x_{3}+x_{1} x_{2}
\end{array}\right. \\
& \left\{\begin{array}{l}
d y_{1} / d t=0.4 y_{1}-y_{2} y_{3} \\
d y_{2} / d t=-12 y_{2}+y_{1} y_{3} \\
d y_{3} / d t=-5 y_{3}+y_{1} y_{2}
\end{array}\right.
\end{aligned}
$$

System (1) and system (2) are chaotic systems. Their chaotic attractors are show as Fig.1 and Fig.2. They are different chaotic system [15].

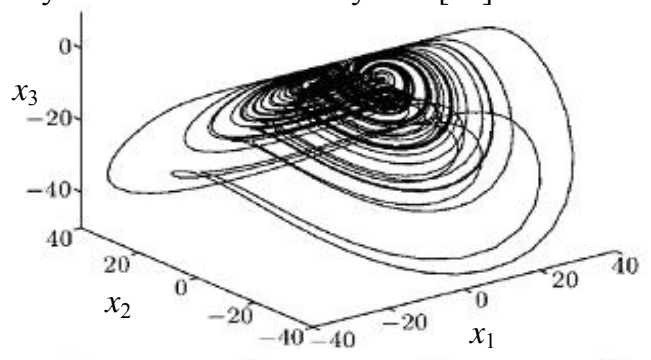

Fig.1 Chaotic attractor of system (1).

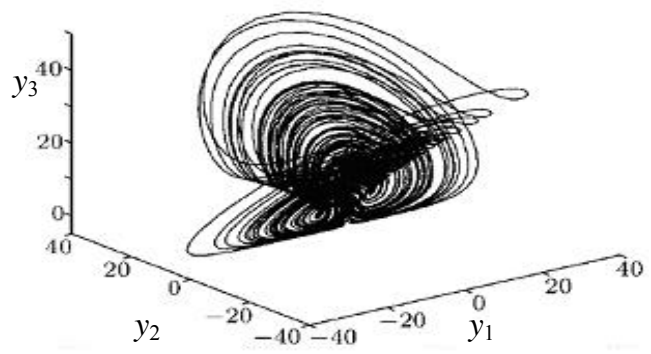

Fig.2 Chaotic attractor of system (2).

In order to realize the chaotic synchronization between system (1) and system (2), we take system (1) as drive system, and system (2) as response system. Therefore, we define the drive and response system as 
follows.

$$
\begin{gathered}
\left\{\begin{array}{l}
d x_{1} / d t=20 x_{1} / 7-x_{2} x_{3} \\
d x_{2} / d t=-10 x_{2}+x_{1} x_{3} \\
d x_{3} / d t=-4 x_{3}+x_{1} x_{2}
\end{array}\right. \\
\left\{\begin{array}{l}
d y_{1} / d t=0.4 y_{1}-y_{2} y_{3}+u_{1}(t) \\
d y_{2} / d t=-12 y_{2}+y_{1} y_{3}+u_{2}(t) \\
d y_{3} / d t=-5 y_{3}+y_{1} y_{2}+u_{3}(t)
\end{array}\right.
\end{gathered}
$$

Where, we have introduced three control function $u_{1}(t), u_{2}(t)$ and $u_{3}(t)$ for system (2). Our goal is to determine the control function $u_{1}(t), u_{2}(t)$ and $u_{3}(t)$, and realize the synchronization between system (3) and system (4). Furthermore, we need not cancel all the nonlinear information in system (4), and will preserves partial nonlinear information for response system (4). This is different from many investigated results [14-15].

Now, we define the error $e_{i}=y_{i}-x_{i}, i=1,2,3$ for system (3) and system (4). We can obtain the follow theorem.

Theorem 1: if we choose

$\mathbf{u}(t)=\left[\begin{array}{ccc}17.2 / 7 & 0 & 0 \\ 0 & 2 & 0 \\ 0 & 0 & 1\end{array}\right]\left[\begin{array}{c}x_{1} \\ x_{2} \\ x_{3}\end{array}\right]-\left[\begin{array}{ccc}0 & -x_{3} & -x_{2} \\ x_{3} & 0 & x_{1} \\ x_{2} & x_{1} & 0\end{array}\right]\left[\begin{array}{c}e_{1} \\ e_{2} \\ e_{3}\end{array}\right]+\mathbf{C}\left[\begin{array}{l}e_{1} \\ e_{2} \\ e_{3}\end{array}\right]$, and all the eigenvaules of matrix $\left[\begin{array}{ccc}0.4 & 0 & 0 \\ 0 & -12 & 0 \\ 0 & 0 & -5\end{array}\right]+\mathbf{C}$ have negative real part, then the chaotic synchronization of system (4) and system (3) can be achieved. Where $\mathbf{C}$ is a suitable constant matrix.

According to the above, the feedback function $\mathbf{u}(t)$ don't consist the nonlinear term of system (2), so we preserves partial nonlinear information for response system (4).

Proof

Subtracting Eq. (3) from Eq. (4), we can obtain the follow (5).

$$
\left.\left[\begin{array}{l}
\dot{e}_{1} \\
\dot{e}_{2} \\
\dot{e}_{3}
\end{array}\right]=\left(\begin{array}{ccc}
0.4 & 0 & 0 \\
0 & -12 & 0 \\
0 & 0 & -5
\end{array}\right]+\mathbf{C}\right)\left[\begin{array}{l}
e_{1} \\
e_{2} \\
e_{3}
\end{array}\right]+\left[\begin{array}{l}
-e_{2} e_{3} \\
e_{1} e_{3} \\
e_{1} e_{2}
\end{array}\right]
$$

Certainly, $e_{i}=y_{i}-x_{i}=0, i=1,2,3$ is one fixed point of error dynamical system (5). The Jacobi matrix of (5) at this fixed point is as follow.

$$
\mathbf{J}=\left[\begin{array}{ccc}
0.4 & 0 & 0 \\
0 & -12 & 0 \\
0 & 0 & -5
\end{array}\right]+\mathbf{C}
$$

Because all the eigenvaules of matrix $\left[\begin{array}{ccc}0.4 & 0 & 0 \\ 0 & -12 & 0 \\ 0 & 0 & -5\end{array}\right]+\mathbf{C}$ have negative real part, so the fixed point $e_{i}=y_{i}-x_{i}=0, i=1,2,3$ is asymptotical stable. So, the zero solution of Eq. (5) is asymptotical stable. Namely, $\lim _{t \rightarrow+\infty}\left(y_{i}-x_{i}\right)=0(i=1,2,3)$. Therefore, the chaotic synchronization of system (4) and system (3) can be achieved.

If we take system (2) as drive system, and system (1) as response system. Therefore, we define the drive system (8) and response system (7) as follows.

$$
\begin{gathered}
\left\{\begin{array}{l}
d x_{1} / d t=20 x_{1} / 7-x_{2} x_{3}+w_{1}(t) \\
d x_{2} / d t=-10 x_{2}+x_{1} x_{3}+w_{2}(t) \\
d x_{3} / d t=-4 x_{3}+x_{1} x_{2}+w_{3}(t)
\end{array}\right. \\
\left\{\begin{array}{l}
d y_{1} / d t=0.4 y_{1}-y_{2} y_{3} \\
d y_{2} / d t=-12 y_{2}+y_{1} y_{3} \\
d y_{3} / d t=-5 y_{3}+y_{1} y_{2}
\end{array}\right.
\end{gathered}
$$

Our goal is to determine the control function $w_{1}(t), w_{2}(t)$ and $w_{3}(t)$, and realize the synchronization between system (7) and system (8).

Now, we define the error $\mathbf{e}=\mathbf{X}-\mathbf{Y}\left(e_{i}=x_{i}-y_{i}, i=1,2,3\right)$ for system (7) and system (8). We can obtain the follow theorem.

Theorem 2: if we choose

$\mathbf{w}(t)=\left[\begin{array}{ccc}-17.2 / 7 & 0 & 0 \\ 0 & -2 & 0 \\ 0 & 0 & -1\end{array}\right]\left[\begin{array}{l}y_{1} \\ y_{2} \\ y_{3}\end{array}\right]-\left[\begin{array}{ccc}0 & -y_{3} & -y_{2} \\ y_{3} & 0 & y_{1} \\ y_{2} & y_{1} & 0\end{array}\right]\left[\begin{array}{c}e_{1} \\ e_{2} \\ e_{3}\end{array}\right]+\mathbf{D}\left[\begin{array}{l}e_{1} \\ e_{2} \\ e_{3}\end{array}\right]$, and all the eigenvaules of matrix $\left[\begin{array}{ccc}20 / 7 & 0 & 0 \\ 0 & -10 & 0 \\ 0 & 0 & -4\end{array}\right]+D$ have negative real part, then the chaotic synchronization of system (7) and system (8) can be achieved. Where $\mathbf{D}$ is a suitable constant matrix.

Proof

Subtracting Eq. (8) from Eq. (7), we can obtain the follow (9).

$$
\left.\left[\begin{array}{l}
\dot{e}_{1} \\
\dot{e}_{2} \\
\dot{e}_{3}
\end{array}\right]=\left(\begin{array}{ccc}
20 / 7 & 0 & 0 \\
0 & -10 & 0 \\
0 & 0 & -4
\end{array}\right]+\mathbf{D}\right)\left[\begin{array}{l}
e_{1} \\
e_{2} \\
e_{3}
\end{array}\right]+\left[\begin{array}{l}
-e_{2} e_{3} \\
e_{1} e_{3} \\
e_{1} e_{2}
\end{array}\right]
$$

Certainly, $e_{i}=y_{i}-x_{i}=0, i=1,2,3$ is one fixed point of error dynamical system (9). The Jacobi matrix of (9) at this fixed point is as follow.

$$
\mathbf{J}=\left[\begin{array}{ccc}
20 / 7 & 0 & 0 \\
0 & -10 & 0 \\
0 & 0 & -4
\end{array}\right]+\mathbf{D}
$$

Because all the eigenvaules of matrix $\left[\begin{array}{ccc}20 / 7 & 0 & 0 \\ 0 & -10 & 0 \\ 0 & 0 & -4\end{array}\right]+$ D have negative real part, so this
fixed point is asymptotical stable. Namely, 
$\lim \left(x_{i}-y_{i}\right)=0(i=1,2,3)$. Therefore, the chaotic synchronization of system (7) and system (8) can be achieved.

According to Theorem 1, if we choose suitable constant matrix $\mathbf{C}$, then the chaotic synchronization of system (4) and system (3) can be achieved. According to Theorem 2, if we choose suitable constant matrix D, then the chaotic synchronization of system (7) and system (8) can be achieved.

\section{Simulation results}

According to the above theorem, our goal is choose suitable constant matrix $\mathbf{C}$ or matrix D. Now, we take some case for example.

\subsection{System (1) as drive system}

According to the above theorem, we need all the eigenvaules of matrix $\left[\begin{array}{ccc}0.4 & 0 & 0 \\ 0 & -12 & 0 \\ 0 & 0 & -5\end{array}\right]+\mathbf{C}$ have negative real part. So, there are many constant matrix $\mathbf{C}$. Therefore, we can realize the chaotic synchronization between system (4) and system (3) easily.

For example, we can choose $\mathbf{C}=\left[\begin{array}{ccc}-0.4 & 1 & 0 \\ 0 & 12 & 1 \\ -8 & -20 & 0\end{array}\right]$,

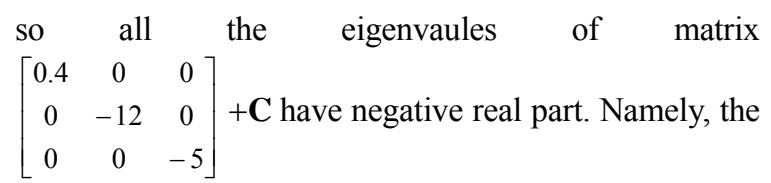

chaotic synchronization of system (4) and system (3) can be achieved. The simulation result is shown as Fig.3. Fig.4 shows the simulation result for $\mathbf{C}=$ $\left[\begin{array}{ccc}-0.4 & 1 & 0 \\ 0 & 12 & 1 \\ -10 & -20 & -25\end{array}\right]$. Where the initial conditions are $\left(y_{1}(0), y_{2}(0), y_{3}(0)\right)=(4,6,1) \quad$ and $\left(x_{1}(0), x_{2}(0), x_{3}(0)\right)=(3,3,-1)$, and $\varepsilon=\left(\sum_{i=1}^{3}\left(y_{i}-x_{i}\right)^{2}\right)^{1 / 2}$.

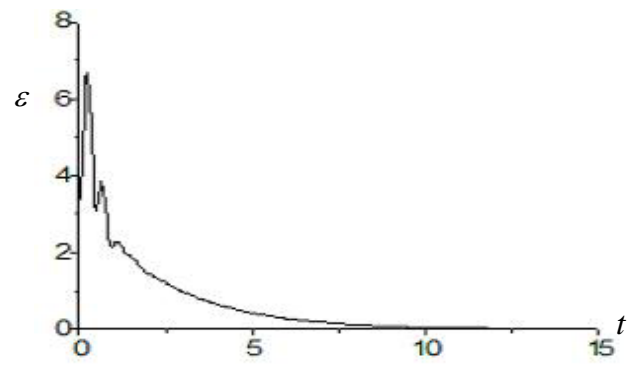

Fig.3: Chaotic synchronization simulation result between system (4) and system (3).

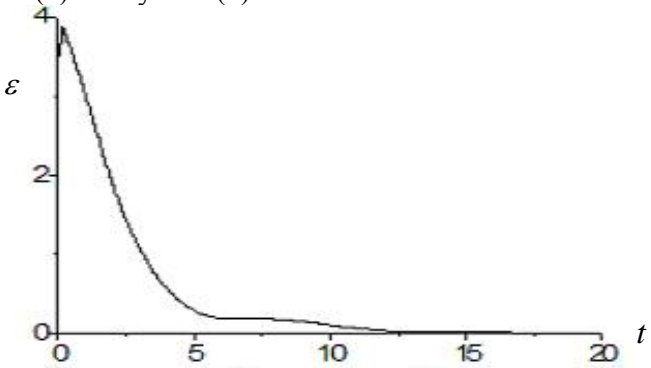

Fig.4: Chaotic synchronization simulation result between system (4) and system (3).

\subsection{System (2) as drive system}

According to the above theorem, we need all the eigenvaules of matrix $\left[\begin{array}{ccc}20 / 7 & 0 & 0 \\ 0 & -10 & 0 \\ 0 & 0 & -4\end{array}\right]+\mathbf{D}$ have negative real part. So, there are many constant matrix D . Therefore, we can realize the chaotic synchronization between system (7) and system (8) easily.

For example, we can choose $\mathbf{D}=\left[\begin{array}{ccc}-20 / 7 & 1 & 0 \\ 0 & 10 & 1 \\ -5 & -10 & -1\end{array}\right]$,

so all the eigenvaules of matrix

$\left[\begin{array}{ccc}20 / 7 & 0 & 0 \\ 0 & -10 & 0 \\ 0 & 0 & -4\end{array}\right]+$ D have negative real part. Namely,

the chaotic synchronization of system (7) and system (8) can be achieved. The simulation result is shown as Fig.5. Fig.6 shows the simulation result for $\mathbf{D}=$ $\left[\begin{array}{ccc}-20 / 7 & 1 & 0 \\ 0 & 10 & 1 \\ -1 & -10 & -1\end{array}\right]$. Where the initial conditions are

$\left(x_{1}(0), x_{2}(0), x_{3}(0)\right)=(1,3,-1) \quad$ and $\left(y_{1}(0), y_{2}(0), y_{3}(0)\right)=(4,8,7)$. 


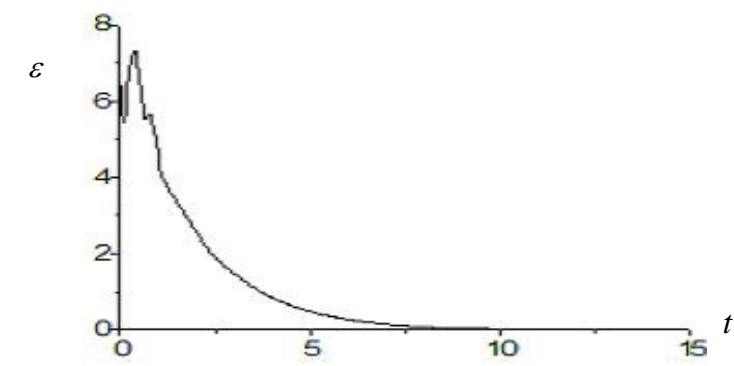

Fig.5: Chaotic synchronization simulation result between system (7) and system (8).

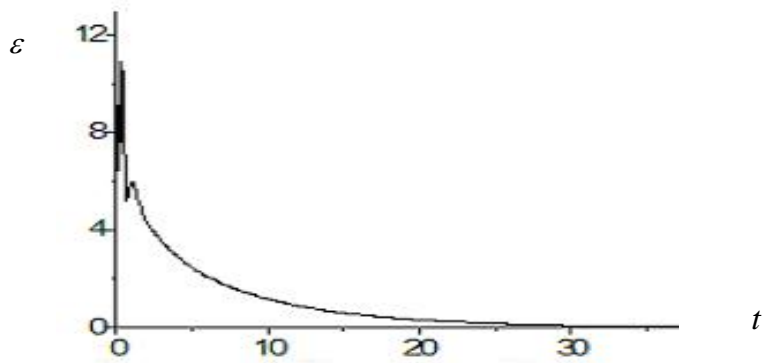

Fig.6: Chaotic synchronization simulation result between system (7) and system (8).

\section{Conclusions}

One generalized synchronization method for two new different chaotic systems is established, and the mathematical proof of this method is provided. Our method doesn't cancel all nonlinear information of response system, and we preserve partial nonlinear information for response system. This is different with many investigated results. Theoretical analyze and simulation results show that the method in this paper is effective.

\section{Acknowledgement}

This work is supported by Chongqing education committee Foundation of China (Grant No.KJ070502)

\section{References}

[1] L.M. Pecora, T.L. Carroll, Synchronization in chaotic systems, Phys. Rev. Lett., 8:821-826, 1990.

[2] E. Ott, C. Grebogi, J.A. Yorke, Driving systems with chaotic signals, Phys. Rev., 11:2374-2379, 1991.

[3] J.C. Sproott, A new class of chaotic circuit, Phys. Rev. Lett. A, 266:19-24, 2000.
[4] S.Y. Zhong, Z.G. Zhou, Q.D. Lian, Passive control of chaotic system with multiple strange attractors, Chinese Physics, 10: 2266-2270, 2006.

[5] S.L Yan, S.B. Wu, H.G. Pang et .al., Studies on injected-feedback and dynamic controls of chaotic systems, Acta Phys. Sin., 3:428-433, 2001.

[6] G. Chen, X. Dong, From Chaos to Order: Methodologies, Perspectives and Applications, Singapore: World Scientific, 1998.

[7] M.P. Kennedy, G.K Columban, Communications using chaos performance bounds for correlation receivers, IEEE Trans. Circuits Syst I :Fundam. Theory Appl., 47:1673-1683,2000.

[8] P. Zhou, Observers for a class of 3D continuous chaotic systems, Acta Phys. Sin., 5:1108$1111,2003$.

[9] X.S. Yang, Q.D. Li, Multiple-scrolls chaotic attractor and circuit implementation, Elec. Lett.,39:1306-1307, 2003.

[10] P. Zhou, X.H. Luo, H.Y. Chen, A new chaotic circuit and its experimental results, Acta Phys. Sin., 11:5048-5052, 2005.

[11] X.S. Yang, Q.D. Li, Chaos generator via Wienbridge oscillator, Int. J. Bifur. Chaos, 5:11591162, 2002.

[12] X.D. Zhang, Z. P Li., Anti-control and generalized synchronization of chaos for a kind of n-dimensional linear differential system, Commun. Theor. Phys., 3:461-472, 2006.

[13] J. Chen, Z.R. Liu, A method of controlling synchronization in different system, Chinese Phys. Lett., 9:1441-1443, 2003.

[14] H.N. Agiza and M.T. Yassen, Mathematics synchronization of Rossler and Chen chaotic dynamical systems using active control, Physics Letter, 278:191-197,2001.

[15] F.Y Sun, Global chaos synchronization between two new chaotic systems via active control, Chinese Phys. Lett., 1:32-34, 2006. 